\title{
Relative Entropy Method for Regional Allocation of Water Pollution Loading
}

\author{
Ju-liang Jin ${ }^{1}$, Yi-ming Wei ${ }^{2}$, Lin-yan Jing ${ }^{1}$, and Yan Guo ${ }^{1}$ \\ ${ }^{1}$ College of Civil Engineering, Hefei University of Technology, Hefei, 230009, China \\ ${ }^{2}$ Center for Energy and Environmental Policy Research, Institute of Policy and Management, \\ Chinese Academy of Sciences, Beijing 100080, China
}

JINJL66@126. com

\begin{abstract}
Regional allocation of water pollution loading is an important measure for achieving general quality and quantity control, which key problem is allocating right weights to sub-regions. Information entropy method (IEM) is proposed to mine the objective variation information of sub-regions and experience information of decision makers during the allocation process. The research results show that IEM is concise and universal, so it can be widely applied to theory and practice of various systems engineering applications.
\end{abstract}

Keywords: Allocation weighting, Analytic hierarchy process, Principle of minimum relative information entropy, Water pollution loads.

\section{Introduction}

Regional allocation of water pollution loading is important for achieving water controls, ensuring a sustainable utilization of a water-based environment, and supporting regional sustainable development [1]. Extant research methods are mostly based on the principle of economic optimization, the principle of equitableness, and the belief of both economic optimization and equitableness [2]. Using Delphi techniques and an Analytic Hierarchy Process (AHP), Li et al. argued for an allocation method of permissible regional water pollution loads [2], which key problem is how to conveniently determine allocation weights of sub-regions using objective information of sub-regions and experience information of decision-makers. Therefore, in this paper a method based on the relative information entropy principle (IEM) is discussed for allocating permissible polluted water at regional levels.

\section{Relative Information Entropy Method for Regional Allocation of Water Pollution Loading}

The establishment of IEM requires three steps, as follows.

Step 1: Establishing the hierarchy structure allocation model of region permissible water pollution loading. In order not to lose universality, the hierarchy structure 
model is constituted of, from top-down, goal level A, criterion level B, index level C, and evaluated object level D.

Step 2: Determining the allocation weights of each sub-regions' permissible water pollution loading, $y_{k}, k=1-n_{d}$, where $n_{d}$ is the number of sub-regions. Because the ninepoint ratio-scale judgment matrix of AHP does not satisfy both linguistic and applied conventions, we use the fuzzy complementary judgment matrix that conforms to Chinese linguistic and cultural norms [3]. Evaluation is made of the importance of each element in B-D levels for upper levels, and to establish judgment matrixes of B, $\mathrm{C}$ and D level as follows: $\boldsymbol{A}=\left\{a_{i j} \mid i, j=1-n_{b}\right\}, \boldsymbol{B}^{k}=\left\{b_{i j}^{k} \mid i, j=1-n_{c} \bullet k=1-n_{b}\right\}$, and $\boldsymbol{C}^{k}=\left\{c_{i j}^{k} \mid i\right.$, $\left.j=1-n_{d} ; k=1-n_{c}\right\}$ respectively. Where $n_{b}, n_{c}$ and $n_{d}$ is the number of element $\mathrm{B}, \mathrm{C}$ and D level, respectively. For brevity, $\boldsymbol{A}$ is described as an example to explain the process of computing weighting. According to Eq. (1), $\boldsymbol{A}$ can be changed into positive reciprocal judgment matrix $\boldsymbol{E}$ with nine-point ratio scale:

$$
e_{i j}=\left\{\begin{array}{c}
\left(b_{m}-1\right)\left(r_{i}-r_{j}\right) /\left(r_{\max }-r_{\min }\right)+1, r_{i} \geq r_{j} \\
1 /\left[\left(b_{m}-1\right)\left(r_{i}-r_{j}\right) /\left(r_{\max }-r_{\min }\right)+1\right], r_{i}<r_{j}
\end{array}\right.
$$

Where priority index $r_{i}=\sum_{j=1}^{n_{b}} a_{i j}, i=1-n_{b} ; r_{\min }$ and $r_{\max }$ are the minimal and maximal value in $\left\{r_{i}\right\}$ respectively; $b_{m}=r_{\max } / r_{\text {min. }}$. Suppose the single priority weight value of each element of $\boldsymbol{E}$ is $w_{k}, \quad k=1-n_{b}$. If $\boldsymbol{E}$ satisfies the consistency condition, then

$$
\sum_{j=1}^{n_{b}}\left(e_{i j} w_{j}\right) / n_{b}=w_{i} \quad\left(i=1-n_{b}\right)
$$

There is no general and precise scale to measure the priority of elements in the system, AHP only requires $\boldsymbol{E}$ to satisfy consistency in order to adapt to different complex useable systems. Therefore the left section of Eq. (2) can be considered as some prior probability distribution of the random variable which is relative to $\boldsymbol{E}$, $\left\{w_{k} \mid k=1-n_{b}\right\}$ can be computed according to the relative entropy principle [4].

$$
\begin{gathered}
\min Q=\sum_{i=1}^{n_{b}} w_{i}\left[\ln w_{i}-\ln \sum_{j=1}^{n_{b}}\left(e_{i j} w_{j} / n_{b}\right)\right] \\
\text { s.t. } \sum_{i=1}^{n_{b}} w_{i}=1, w_{i}>0, i=1-n_{b}
\end{gathered}
$$

Using the Lagrange multipliers method to solve Eq. (3) and Eq. (4), we obtain

$$
w_{i}=\sum_{j=1}^{n_{b}}\left(e_{i j} w_{j}\right) / \sum_{i=1}^{n_{b}} \sum_{j=1}^{n_{b}}\left(e_{i j} w_{j}\right) \quad\left(i=1-n_{b}\right)
$$


So the iterative computing method can be used to compute $w_{i}$ as follows:

$$
w_{i}^{t}=\sum_{j=1}^{n_{b}}\left(e_{i j} w_{j}^{t-1}\right) / \sum_{i=1}^{n_{b}} \sum_{j=1}^{n_{b}}\left(e_{i j} w_{j}^{t-1}\right) \quad\left(i=1-n_{b} ; t=1,2, \ldots\right)
$$

Where $w_{i}^{t}$ is the $t$ th iterative computed weight of the $i$ th element. According to Eq. (2), Jin et al. defined the consistency index coefficient (CIC) of $\boldsymbol{E}$ as follows [5]

$$
\operatorname{CIC}\left(n_{b}\right)=\sum_{i=1}^{n_{b}}\left|\sum_{j=1}^{n_{b}}\left(e_{i j} w_{j}\right)-n_{b} w_{i}\right| / n_{b}
$$

When $C I C<0.10, \boldsymbol{E}$ can be considered as satisfying consistency. In a similar way, we can calculate the weight of each element of each judgment matrix of $\mathrm{C}$ level $w_{j}^{i}(j=1$ $\left.n_{c}, i=1-n_{b}\right)$, and that of D level $w_{k}^{j}\left(k=1-n_{d}, j=1-n_{c}\right)$, then find the total weight of each element of $\mathrm{C}$ level to the goal level A:

$$
w_{j}^{A}=\sum_{i=1}^{n_{b}} w_{i} w_{j}^{i} \quad\left(j=1-n_{c}\right)
$$

The allocation weight of permissible water pollution loads of each sub-region is

$$
y_{k}=\sum_{j=1}^{n_{c}} \sum_{i=1}^{n_{b}} w_{i} w_{j}^{i} w_{k}^{j} \quad\left(k=1-n_{d}\right)
$$

Step 3: Allocating reasonable regional permissible water pollution loading for each sub-region according to the allocation weights of each sub-region $y_{k}$.

\section{Case Study}

A river channel-type reservoir receives the domestic sewage and industrial wastewater from four cities in the drainage area. According to the goal of municipal water-quality planning, we know that the permissible total water pollution loading of chemical oxygen demanded organic pollutant (COD) of the water body is 35 000t/a [2]. The goal level $\mathrm{A}$ of the hierarchy structure model is the allocation goal. $\mathrm{B}_{1}, \mathrm{~B}_{2}, \mathrm{~B}_{3}$ and $\mathrm{B}_{4}$ is social, economic, environmental, and technological \& managing criterion respectively. In the criterion level $\mathrm{C}, \mathrm{C}_{1}$ is the proportion of non-farm population, $\mathrm{C}_{2}$ is the proportion of tertiary-industry employees, $\mathrm{C}_{3}$ is the unemployment rate of urban workers and staff, $\mathrm{C}_{4}$ is the medical and health condition level, $\mathrm{C}_{5}$ is the social-culture level, $\mathrm{C}_{6}$ is per capita gross domestic product, $\mathrm{C}_{7}$ is per capita revenue of the local government, $\mathrm{C}_{8}$ is the efficiency of social labor, $\mathrm{C}_{9}$ is the area per capita income level, $\mathrm{C}_{10}$ is the area per capita consuming level, $C_{11}$ is the utilization and protection ratio of water resource, $\mathrm{C}_{12}$ is the utilization ratio of carrying capacity of water environment, $\mathrm{C}_{13}$ is wastewater discharge of output of ten thousand yuan, $\mathrm{C}_{14}$ is $\mathrm{COD}$ discharge of output of ten thousand yuan, $C_{15}$ is the urban sewage treatment rate, $C_{16}$ 
is the industrial wastewater increasing to the standard rate, $\mathrm{C}_{17}$ is the wastewater reuse rate, and $\mathrm{C}_{18}$ is advances in science and technology contribution rate. In the evaluated object level $\mathrm{D}, \mathrm{D}_{1}, \mathrm{D}_{2}, \mathrm{D}_{3}$ and $\mathrm{D}_{4}$ are the cities, respectively. According to the Table 1 of reference [2], we obtain the fuzzy complementary judgment matrixes of Level B, $\mathrm{C}$ and D. Using IEM, we can get the weights from criteria 1 to criteria 4 of level $\mathrm{B}$ as $0.0625,0.3125,0.3125$ and 0.3125 respectively, the total weight from $\mathrm{C} 1$ to $\mathrm{C} 18$ relative to the goal of level $\mathrm{A}$ as $0.0074,0.0074,0.0236,0.0074,0.0165,0.1063$, $0.1063,0.0512,0.0243,0.0243,0.1563,0.1563,0.0995,0.0995,0.0403,0.0403$, 0.0164 and 0.0164 respectively, the allocation weights from city 1 to city 4 as 0.178 , $0.411,0.186$ and 0.225 respectively, and the permissible water pollution loading from city 1 to 4 as $6230,14385,6510$ and 7875 t/a respectively. The above results are corresponds to those in [2]. According to the evaluated index values of each city in the Table 1 in [2], comparatively speaking, each index value of city 2 is clearly superior to that of the other three cities. Additionally, most of the indexes of city 4 are superior to those of city 1 and city 3 . Each index value of city 1 is noticeably inferior to that of the other three cities.

\section{References}

1. Gren IM, Folmer H: Cooperation with Respect to Cleaning of an International Water Body with Stochastic Environmental Damage: the Case of the Baltic Sea. Ecological Economics, (47) 33-42 (2003)

2. Ru-zhong Li, Jia-quan Wang, Jia-zhong Qian: Delphi-AHP Method for Allocation of Waste Loads in a Region. Journal of Harbin of Technology, 37(1) 84-88 (2005)

3. Ming Yao: Fuzzy Information Processing Technology with Computer. Shanghai: Science and Technology Press of Shanghai (1999)

4. Xue-long Zhu: Foundation of Application Information Theory. Beijing: Tsinghua University Press (2001)

5. Ju-liang Jin, Yi-ming Wei, Qiang Fu, et al: Accelerating Genetic Algorithm for Computing Rank Weights in Analytic Hierarchy Process. Systems Engineering-Theory \& Practice, 22(11) 39-43 (2002) 